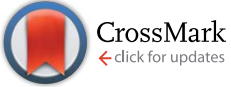

Cite this: RSC Adv., 2017, 7, 8879

Received 17th November 2016 Accepted 18th January 2017

DOI: 10.1039/c6ra26917f

www.rsc.org/advances

\title{
Iron-nitrogen co-doped hierarchically mesoporous carbon spheres as highly efficient electrocatalysts for the oxygen reduction reaction $\uparrow$
}

\author{
You-Lin Liu, + Xue-Yan Xu, $\$$ Cheng-Xiang Shi, Xin-Wei Ye, Ping-Chuan Sun \\ and Tie-Hong Chen*
}

By using hierarchically mesoporous silica spheres as hard template, 2-aminopyridine and $\mathrm{FeCl}_{3}$ as carbon, nitrogen and iron sources, respectively, iron and nitrogen co-doped hierarchically mesoporous carbon spheres (Fe-N-CS) were successfully prepared. The sample Fe-N-CS-900 obtained at a carbonization temperature of $900{ }^{\circ} \mathrm{C}$ exhibited a highly efficient electrocatalytic activity with positive half-wave potential $(-0.11 \mathrm{~V})$, high limiting current density $\left(-4.79 \mathrm{~mA} \mathrm{~cm}^{-2}\right)$ and high selectivity (electron transfer number around 4) for the oxygen reduction reaction (ORR) in alkaline media. Moreover, Fe-N-CS-900 shows higher stability and better methanol tolerance in comparison to commercial Pt/C catalyst in both alkaline and acidic media. Its highly efficient ORR activity could be ascribed to its high specific surface area, unique porous structure and homogeneous distribution of $\mathrm{Fe}-\mathrm{N}_{x}$ active sites formed during pyrolysis.

\section{Introduction}

The oxygen reduction reaction (ORR) plays an important role in various energy storage and conversion process, such as fuel cells and metal-air batteries. ${ }^{1,2}$ Although platinum-based materials are the best ORR catalysts, ${ }^{3,4}$ they suffer from slow reduction kinetics, high cost, low stability and the crossover effect, which are drawbacks for large-scale commercialization. Therefore, exploring non-precious metal (NPM) $)^{5-10}$ and metal-free ${ }^{11-15}$ catalysts with excellent ORR activity and high stability has been attracting extensive interest. Various nitrogen-doped carbons $\mathrm{s}^{16-19}$ and carbon-supported transition metal oxides (Fe, $\mathrm{Co}, \mathrm{Mn})^{20-24}$ have been reported, and these catalysts exhibited an obviously high ORR activity in alkaline media. Unfortunately, in acidic solution most of the nitrogen doped carbon materials had little electrocatalytic activity and lower stability due to the protonation of doped nitrogen active sites, ${ }^{25,26}$ and carbon-supported transition metal oxide catalysts could not be used in acidic media.

Recently, nitrogen doping and transitional metal-contained carbon materials, particularly, $\mathrm{Fe}$ (or $\mathrm{Co}$ ) $-\mathrm{N}_{x} / \mathrm{C}$ have been recognized as the most promising NPM catalysts for ORR in

Institute of New Catalytic Materials Science, School of Materials Science and Engineering, Key Laboratory of Advanced Energy Materials Chemistry (MOE), Collaborative Innovation Centre of Chemical Science and Engineering (Tianjin), Nankai University, Tianjin 300350, PR China.E-mail: chenth@nankai.edu.cn

$\dagger$ Electronic supplementary information (ESI) available. See DOI: 10.1039/c6ra26917f

\$ Contributed equally in this work. both alkaline and acidic media. ${ }^{27-31}$ It is found that Fe (or Co)$\mathrm{N}_{x}$ sites are attributed to the main active sites for ORR. Thus, various methods have being used to improve active sites density of $\mathrm{M}-\mathrm{N}_{x} / \mathrm{C}$ materials. Traditionally, $\mathrm{M}-\mathrm{N}_{x} / \mathrm{C}$ catalysts are prepared by the direct pyrolysis of the mixture of nitrogen precursor, transitional metal and carbon precursor. However this method frequently fails to control the contribution and density of transition metal-nitrogen active sites, leading to relatively poor catalytic performance. ${ }^{32}$

Catalysts with mesoporous structure generally possess high surface area and large pore volume, which not only facilitate the exposure of active sites and benefit the mass transport of intermediate species, but also lead to enhanced electron delivery due to the continuous framework structure. ${ }^{20,33}$ Mesoporous carbon catalysts prepared by template methods, e.g. mesoporous metal-nitrogen-doped carbons, ${ }^{34}$ ordered mesoporous porphyrin-based carbon materials, ${ }^{35,36}$ and ordered mesoporous $\mathrm{Fe}-\mathrm{N}$ carbons,${ }^{37}$ have been reported to enhance the electrocatalytic activity of ORR. Furthermore, hierarchically porous carbon materials co-doped with iron and nitrogen exhibited excellent performance for ORR. ${ }^{38-40}$

Herein we report preparation of iron and nitrogen co-doped hierarchically mesoporous carbon spheres by using hierarchically mesoporous silica spheres as hard template, high nitrogen content monomer (2-aminopyridine) as carbon and nitrogen sources, and $\mathrm{FeCl}_{3}$ as iron source. It is proved that both the Fe$\mathrm{N}_{x}$ active sites and porous structure are very helpful to ORR activity and the carbon spheres exhibited higher stability and better methanol tolerance in comparison to commercial Pt/C catalyst in both alkaline and acidic media. 


\section{Experimental}

Synthesis of hierarchically mesoporous silica sphere template

Hierarchically mesoporous silica spheres (HMS) were synthesized following our previously reported method. ${ }^{41}$ First, $0.55 \mathrm{~g}$ CTAB was dissolved in $25.0 \mathrm{~mL}$ of distilled water, and $3.0 \mathrm{~g}$ PAA (25\%) was added under vigorous stirring at room temperature to obtain a clear solution. Then $2.0 \mathrm{~g}$ ammonia (25\%) was added to the above solution under vigorous stirring. After $20 \mathrm{~min}$ stirring, $2.08 \mathrm{~g}$ tetraethoxysilane (TEOS) was added to the above solution and continued stirring for $15 \mathrm{~min}$, the mixture was transferred into an autoclave, which was put into an oven of $100{ }^{\circ} \mathrm{C}$ for $48 \mathrm{~h}$. The final product was centrifugated, washed with distilled water, and dried at $60{ }^{\circ} \mathrm{C}$. The organic templates were removed by calcination at $550{ }^{\circ} \mathrm{C}$ for $6 \mathrm{~h}$.

\section{Synthesis of porous carbon spheres}

In a typical synthesis of iron-nitrogen co-doped hierarchically mesoporous carbon spheres (denoted as Fe-N-CS-T, T: carbonization temperature), $1.2 \mathrm{~g}$ HMS template were stirred in toluene solution containing $1.43 \mathrm{~g}$ 2-aminopyridine, after stirring for $10 \mathrm{~min}, 3.45 \mathrm{~g}$ ammonium persulphate and $0.82 \mathrm{~g}$ $\mathrm{FeCl}_{3} \cdot 6 \mathrm{H}_{2} \mathrm{O}$ were added to above mixture solution and the resultant mixture were stirred for $12 \mathrm{~h}$ in an ice bath $\left(0-10^{\circ} \mathrm{C}\right)$. This led to polymerization of 2-aminopyridine, after polymerization, the obtained mixture solution was centrifugated and the solid powder was dried at $120^{\circ} \mathrm{C}$ for $12 \mathrm{~h}$, finally the brown solid powder precursors were obtained. Then the precursors were pyrolyzed at different temperature (700-1000 ${ }^{\circ} \mathrm{C}$ ) in a flow of high-purity $\mathrm{N}_{2}$ atmosphere. After removal silica by $\mathrm{HF}$ ( $20 \mathrm{wt} \%$ ) etching, Fe-N-CS-T samples were obtained. For comparison, Fe-free samples (N-CS-T) were obtained without adding $\mathrm{FeCl}_{3}$ during the polymerization process.

\section{Characterization}

Field-emission scanning electron microscopy (FESEM; JEOL, JSM-7500F, $8 \mathrm{kV}$ ) was used to analyze the morphology of the prepared samples. Transmission electron microscopy (TEM) was performed on a Philips Tecnai F20 microscope, working at $200 \mathrm{kV}$. All samples subjected to TEM measurements were dispersed in ethanol ultrasonically and were dropped on copper grids. Raman spectra were examined with a LabRAM HR Raman spectrometer using laser excitation at $\mathbf{5 1 4 . 5} \mathrm{nm}$. Powder X-ray diffraction (XRD) patterns were recorded on a D/max-2500 diffractometer (Rigaku, Japan) using $\mathrm{Cu} \mathrm{K} \alpha$ radiation $(\lambda=$ $1.5406 \AA$ A). XPS spectra were obtained with a Kratos Axis Ultra DLD spectrometer. Nitrogen adsorption and desorption isotherms were measured on a BELSORP-mini II sorption analyser at $77 \mathrm{~K}$.

\section{Electrochemical measurements}

Electrochemical experiments were conducted at room temperature on a CHI660D electrochemical workstation. A threeelectrode system including a glassy carbon rotating disk electrode (RDE) coated with catalysts, a platinum wire auxiliary electrode and a $\mathrm{KCl}(3 \mathrm{M}) \mathrm{Ag} / \mathrm{AgCl}$ reference electrode. For electrode preparation, $2 \mathrm{mg}$ sample was dispersed in $1 \mathrm{~mL}$ of $\mathrm{H}_{2} \mathrm{O}$ by sonication for $30 \mathrm{~min}$ at least. Then $20 \mu \mathrm{L}$ of the resulting homogeneous suspension was carefully dropped with pipette onto a rotation disk electrode (RDE), which was air-dried to allow solvent evaporation. After drying at room temperature, $3 \mu \mathrm{L}$ of $5 \mathrm{wt} \%$ Nafion solution was further dropped to form a protection layer against catalyst detaching from the electrode surface. The catalyst loading was $0.41 \mathrm{mg} \mathrm{cm}^{-2}$ in alkaline media. High-purity $\mathrm{N}_{2}$ or $\mathrm{O}_{2}$ was purged for $30 \mathrm{~min}$ at least before each test. For comparison, the benchmark commercial $\mathrm{Pt} / \mathrm{C}$ (20 wt\% Pt on VXC-72) catalyst was also fabricated into electrodes through similar procedure, with the loading of $62 \mu \mathrm{g}$ Pt per $\mathrm{cm}^{2}$ in alkaline media. For ORR in acidic media, $0.5 \mathrm{M}$ $\mathrm{H}_{2} \mathrm{SO}_{4}$ solution and $0.1 \mathrm{M} \mathrm{HClO}_{4}$ solution were used as electrolytes for Koutecky-Levich analysis of the prepared catalysts $\left(0.62 \mathrm{mg} \mathrm{cm}^{-2}\right)$ and $\mathrm{Pt} / \mathrm{C}$ catalyst ( $41 \mu \mathrm{g}$ Pt per $\left.\mathrm{cm}^{2}\right)$, respectively.

\section{Results and discussion}

The procedure for synthesis of the $\mathrm{Fe}-\mathrm{N}$ co-doped carbon spheres is illustrated in Scheme 1. A high nitrogen content monomer (2-aminopyridine) first entered into the mesopores of silica spheres, and then in situ oxidation polymerization reaction was performed in the mesopores. The obtained precursors polymer within the mesopores of silica template (polymer $/ \mathrm{SiO}_{2}$ ) were dried and then pyrolyzed at different temperature (700$1000{ }^{\circ} \mathrm{C}$ ) under flowing high-purity nitrogen atmosphere, the black power was obtained. Finally, the samples (Fe-N-CS-T) were obtained by removing silica templates.

The morphology and structure of $\mathrm{Fe}-\mathrm{N}-\mathrm{CS}-900$ were characterized by scanning electron microscopy (SEM) and transition electron microscopy (TEM). Fig. 1a and b showed Fe-N-CS-900 was composed of well-defined carbon spheres with rough surface, which successfully copied the morphology of mesoporous template (Fig. S1 $\dagger$ ). TEM (Fig. 1c and d) indicated the carbon spheres were three-dimensional connected porous framework structure. These observations well reflected the geometric characteristic of the original template, suggesting successful preparation of porous carbon spheres.

The porosity of Fe-N-CS- $T$ was analyzed by $\mathrm{N}_{2}$ adsorption isotherms (Fig. 2, S2 and Table S1 $\dagger$ ). Fe-N-CS-900 exhibited a type IV isotherm with three adsorption steps at the relative pressure of $0.01-0.1,0.20-0.70$, and $0.80-0.95$, respectively. The first step corresponded to the micropores within the carbon matrix. The second adsorption gave rise to a pore size distribution at about 2-3 $\mathrm{nm}$ (Fig. 2b), which was ascribed to the

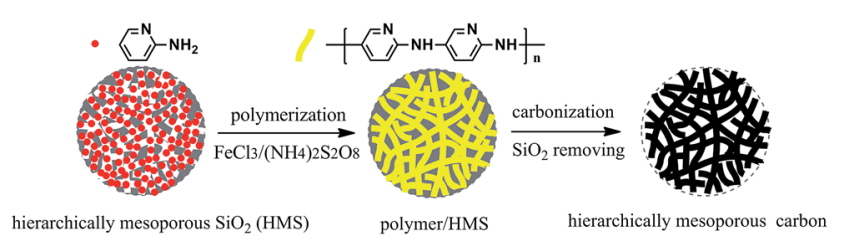

Scheme 1 Illustration of preparation of hierarchically mesoporous carbon spheres. 


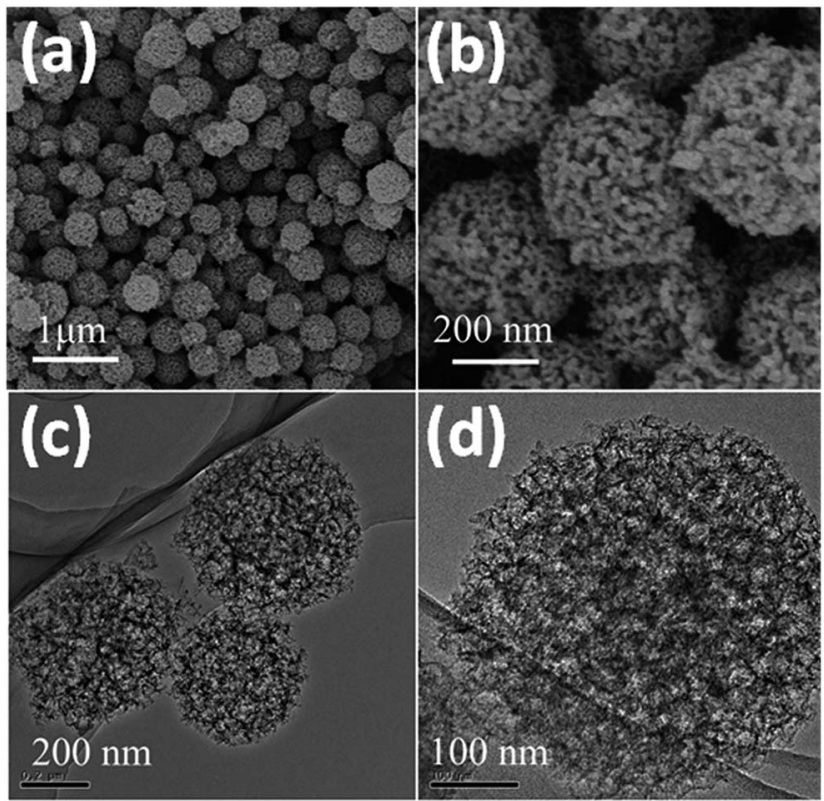

Fig. 1 (a and b) Typical FESEM images of Fe-N-CS-900. (c and d) Typical TEM images of Fe-N-CS-900
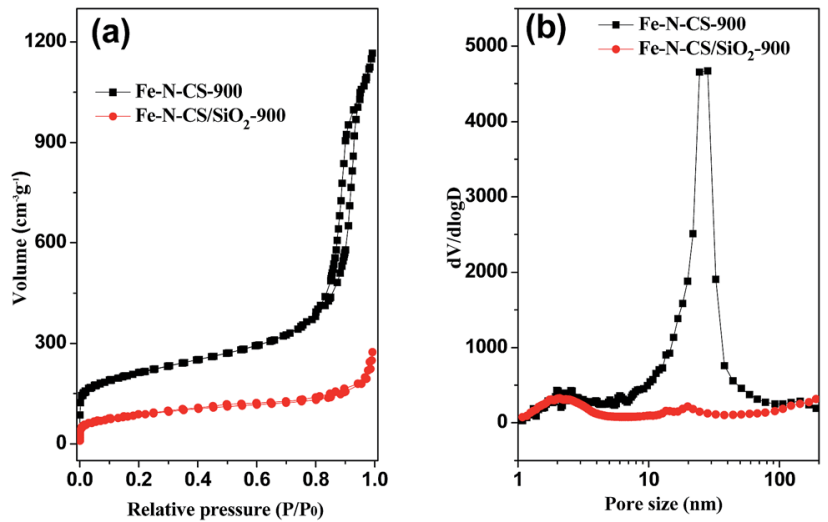

Fig. 2 (a) Nitrogen adsorption-desorption isotherms of Fe-N-CS900, $\mathrm{Fe}-\mathrm{N}-\mathrm{CS} / \mathrm{SiO}_{2}-900$ and (b) their corresponding pore size distribution curve.

small disordered mesopores. The third adsorption step gave rise to a peak at about $25 \mathrm{~nm}$ in the pore size distribution curve, corresponding to larger mesopores (Fig. 2b). Table S1† showed that Fe-N-CS-900 exhibited highest specific surface area of 758 $\mathrm{m}^{2} \mathrm{~g}^{-1}$ and large pore volume of $1.59 \mathrm{~cm}^{3} \mathrm{~g}^{-1}$. In order to investigate the formation of mesopores, the pyrolyzed sample (denoted $\mathrm{Fe}-\mathrm{N}-\mathrm{CS} / \mathrm{SiO}_{2}-900$ ) without removal of the silica template was shown in Fig. 2a, nitrogen adsorption-desorption isotherms of $\mathrm{Fe}-\mathrm{N}-\mathrm{CS} / \mathrm{SiO}_{2}-900$ showed it had little mesoporous structure. This results indicated that most of mesoporous structure of Fe-N-CS-900 was derived from the hierarchically mesoporous silica template.

X-ray photoelectron spectroscopy (XPS) was performed to analyze the content and the chemical state for the surface doping elements in the samples (Fig. 3 and S3†). Fig. S3†
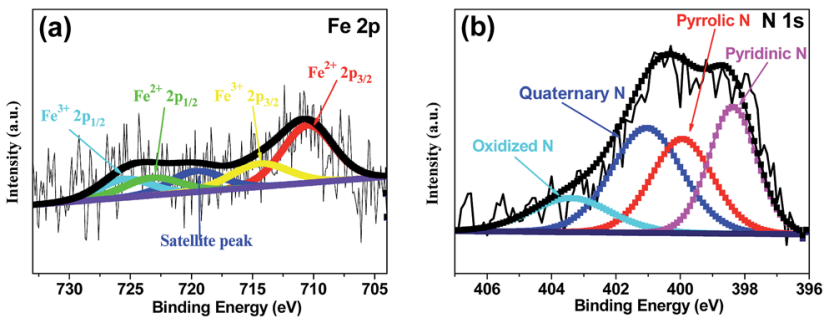

Fig. 3 (a) Fe 2p XPS spectrum and (b) N 1s XPS spectrum of Fe-N-CS900 catalyst.

showed Fe-N-CS-900 had four kinds of elements, carbon, oxygen, nitrogen (4.53 wt\%) and iron (0.83 wt\%). N 1s spectrum of Fe-N-CS-900 catalyst (Fig. 3b) could be divided into four peaks at $398.4 \mathrm{eV}, 400.0 \mathrm{eV}, 401.0 \mathrm{eV}$ and $403.4 \mathrm{eV},{ }^{\mathbf{4 2 , 4 3}}$ which were assigned to pyridinic $\mathrm{N}(27.57 \%)$, pyrrolic $\mathrm{N}(26.39 \%)$, graphitic $\mathrm{N}(33.64 \%)$ and pyridinic $\mathrm{N}-\mathrm{O}(12.41 \%)$. The graphitic $\mathrm{N}$ played a crucial role in oxygen reduction; ${ }^{19}$ the pyridinic $\mathrm{N}$ and pyrrolic $\mathrm{N}$ could serve as metal-coordination sites ${ }^{29,38}$ and these three different nitrogen species were of high content in Fe-N-CS-900, which may lead to high ORR activity. The Fe 2p XPS spectrum of Fe-N-CS-900 (Fig. 3a) was deconvoluted into five different peaks, indicating that iron species in the catalyst were complicated in terms of its metallic state. According to previous reports, ${ }^{28,38}$ the Fe 2 p peaks at $711.3 \mathrm{eV}$ were ascribed to the nitrogen-coordinated metal, which were dominated iron species in $\mathrm{Fe}-\mathrm{N}-\mathrm{CS}-900$ catalyst. In addition, elemental mapping (Fig. 4) imaging revealed that both iron and nitrogen species were homogeneous distributed in the mesoporous carbon spheres. The bright areas in Fig. $4 \mathrm{~b}-\mathrm{d}$ were likely due to differences in carbon thickness in the sample.
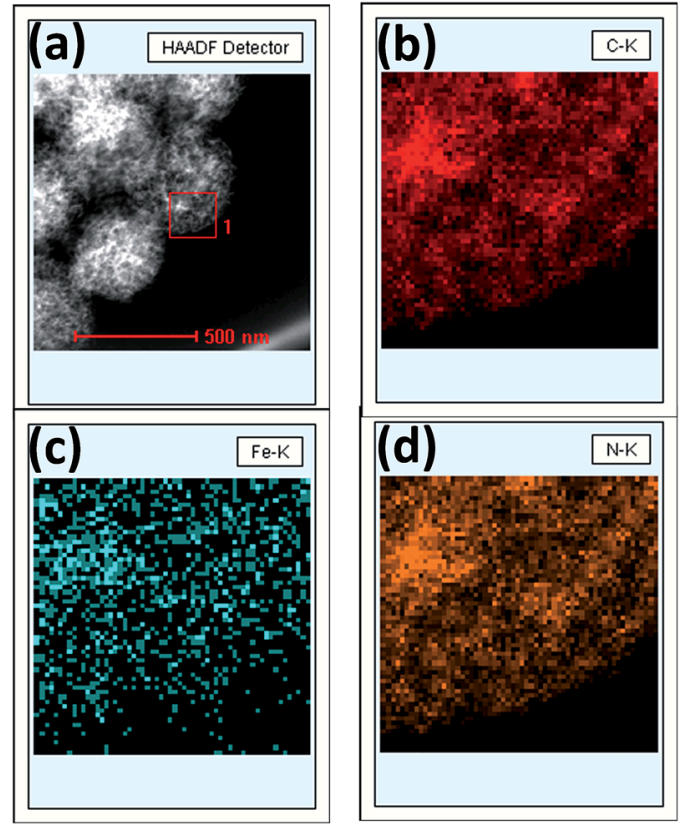

Fig. 4 (a) STEM image of Fe-N-CS-900, (b) carbon-, (c) iron- and (d) nitrogen-elemental mapping of square region 1. 
Although there was an amount of iron species in Fe-N-CS900 sample. We hardly observed any metal nanoparticles in the carbon structure by TEM (Fig. 1d). Power X-ray diffraction pattern (PXRD) further indicated the absence of the crystalline metal or metal oxide nanoparticles and showed only the partially graphitized carbon (Fig. S4 $\dagger$ ). The Raman spectrum (Fig. S5†) showed a typical D-band at $1375 \mathrm{~cm}^{-1}$ and a G-band at $1590 \mathrm{~cm}^{-1}$ indicating co-existence of amorphous and graphitic structures.

Cyclic voltammetry (CV) in $\mathrm{N}_{2}$ and $\mathrm{O}_{2}$-saturated $0.1 \mathrm{M} \mathrm{KOH}$ solution was first evaluated to examine ORR activity for $\mathrm{Fe}-\mathrm{N}-$ CS-T catalysts. Fig. 5a showed that in $\mathrm{N}_{2}$-saturated $\mathrm{KOH}$ solution $\mathrm{CV}$ curve of $\mathrm{Fe}-\mathrm{N}-\mathrm{CS}-900$ exhibited the featureless characteristic, indicating negligible redox reaction and inertness on the carbon surface. In contrast, when the electrolyte was saturated
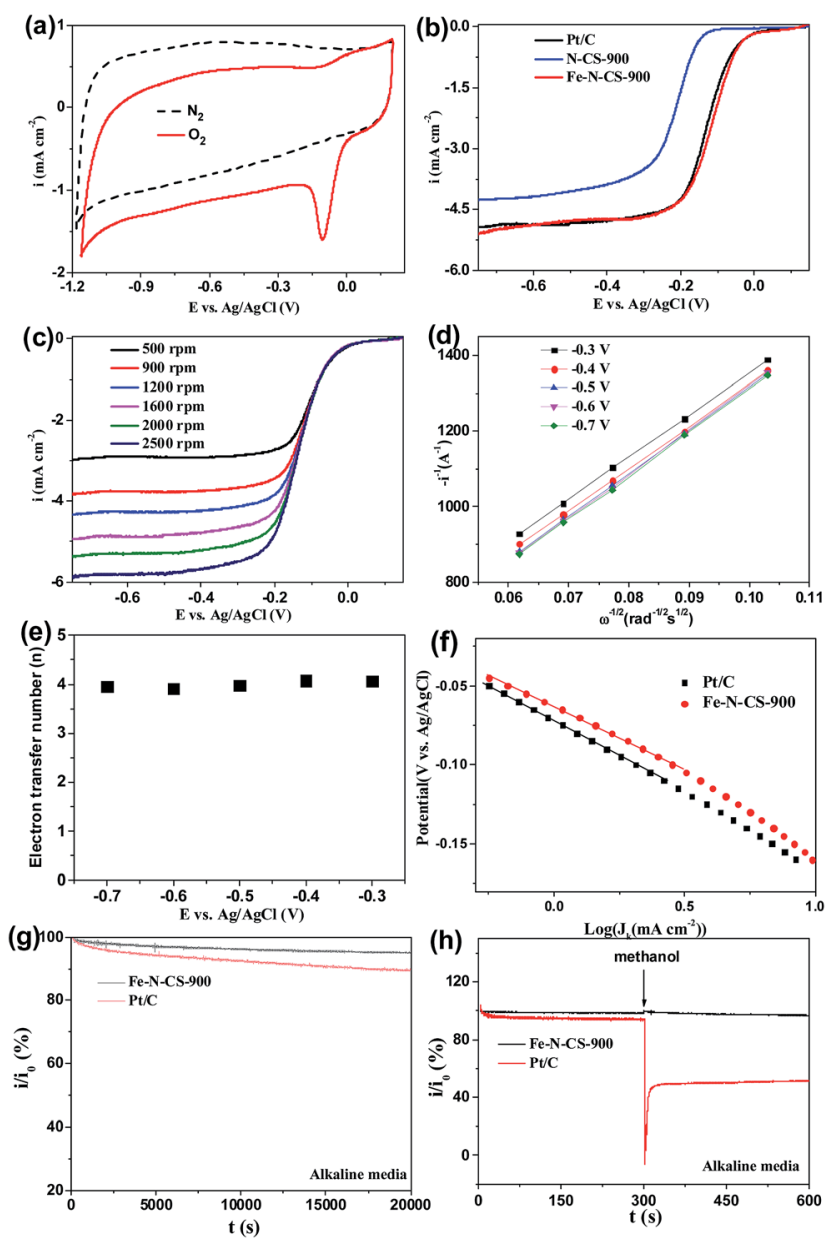

Fig. 5 (a) $\mathrm{CV}$ curves of $\mathrm{Fe}-\mathrm{N}-\mathrm{CS}-900$ catalyst in $\mathrm{N}_{2}$ or $\mathrm{O}_{2}$-saturated $0.1 \mathrm{M} \mathrm{KOH}$ solution. (b) LSV curves of $\mathrm{N}-\mathrm{CS}-900, \mathrm{Fe}-\mathrm{N}-\mathrm{CS}-900$ and $\mathrm{Pt} / \mathrm{C}$ catalysts in $\mathrm{O}_{2}$-saturated $0.1 \mathrm{M} \mathrm{KOH}$ solution. (c) LSV curves for $\mathrm{Fe}-\mathrm{N}-\mathrm{CS}-900$ catalyst at different rotation rate. (d) $\mathrm{K}-\mathrm{L}$ plots and (e) the corresponding electron transfer numbers for $\mathrm{Fe}-\mathrm{N}-\mathrm{CS}-900$ catalyst at different electrode potentials. (f) Tafel plots of Fe-N-CS900 and $\mathrm{Pt}-\mathrm{C}$. The $\mathrm{i} / \mathrm{i}_{0}$ was based on chronoamperometric responses for the ORR on Fe-N-CS-900 catalyst and commercial Pt/C catalyst modified electrodes, (g) in $\mathrm{O}_{2}$-saturated alkaline media for $20000 \mathrm{~s}$, respectively, (h) with $1 \mathrm{M}$ methanol added at around $600 \mathrm{~s}$ at $-0.5 \mathrm{~V}$ in $\mathrm{O}_{2}$-saturated alkaline media. with $\mathrm{O}_{2}$, there is a sharp electrochemical reduction peak (peak potentials at $-0.11 \mathrm{~V}$ ), which was more positive than those of nitrogen-doped carbon spheres, ${ }^{\mathbf{4 4 , 4 5}}$ suggesting the pronounced catalytic activity of $\mathrm{Fe}-\mathrm{N}-\mathrm{C}-900$ catalyst for ORR in an alkaline solution. Fig. S6† showed that the optimal temperature was $900{ }^{\circ} \mathrm{C}$, as revealed by the most positive peak potential and higher peak current density in the CV curves, probably due to an appropriate balance of surface area, active site density, and graphitization degree at this temperature (Table S1, Fig. S4 and $\mathrm{S} 5 \dagger)$. Therefore, the prepared catalysts discussed below were all produced at $900{ }^{\circ} \mathrm{C}$ unless otherwise specified.

To further assess the ORR catalytic activity of the prepared samples, electrocatalytic test were carried out using rotating ring electrodes (RDEs) in an alkaline solution. This was also compared with that of the benchmark commercial Pt/C catalyst $\left(62 \mu \mathrm{g}\right.$ Pt per $\mathrm{cm}^{2}$ ). Fig. 4b showed the typical linear sweep voltammograms (LSVs) at an electrode rotating speed of 1600 revolutions per minute (rpm) and a potential scanning rate of $5 \mathrm{mV} \mathrm{s}^{-1}$. Remarkably, the ORR activity of Fe-N-CS-900 catalyst was close to commercial $\mathrm{Pt} / \mathrm{C}$ catalyst in the whole testing potential region and the half-wave potential for $\mathrm{Fe}-\mathrm{N}-\mathrm{CS}-900$ catalyst $(-0.11 \mathrm{~V})$ was even about $10 \mathrm{mV}$ more positive than that of commercial $\mathrm{Pt} / \mathrm{C}$ catalyst $(-0.12 \mathrm{~V})$. In addition, the limiting current density $\left(-4.79 \mathrm{~mA} \mathrm{~cm}^{-2}\right.$ at $\left.-0.5 \mathrm{~V}\right)$ of $\mathrm{Fe}-\mathrm{N}-\mathrm{CS}-$ 900 catalyst was close to that of commercial $\mathrm{Pt} / \mathrm{C}$ catalyst $(-4.83$ $\mathrm{mA} \mathrm{cm} \mathrm{cm}^{-2}$ at $\left.-0.5 \mathrm{~V}\right)$. These results suggested highly efficient ORR activity of Fe-N-CS-900 in alkaline media. Due to superior ORR activity of Fe-N-CS-900, its ORR kinetic process has also been investigated in alkaline media. As shown in Fig. 5c, the rotation-rate-dependent current-potential curves for Fe-N-CS900 displayed current density increased with higher rotating speeds due to shortened diffusion distance at high rotating speed rate. ${ }^{46}$ The kinetic parameters can be analyzed with the Koutecky-Levich equation:

$$
i^{-1}=i_{\mathrm{k}}^{-1}+i_{\mathrm{L}}^{-1}=(n F k C)^{-1}+\left(0.62 n F C D^{2 / 3} v^{-1 / 6} \omega^{1 / 2}\right)^{-1}
$$

where $i$ is the measured current density, $i_{\mathrm{k}}$ and $i_{\mathrm{L}}$ are the kinetic and diffusion limiting current densities, respectively, $\omega$ is rotation rate of the electrode, $n$ is the electron transfer number, $F$ is the Faraday constant, $C$ is the bulk concentration of $\mathrm{O}_{2}$ dissolved in the electrolyte $\left(1.2 \times 10^{-3} \mathrm{~mol} \mathrm{~L}^{-1}\right), D$ is the diffusion coefficient of $\mathrm{O}_{2}$ in the electrolyte $\left(1.9 \times 10^{-5} \mathrm{~cm}^{-2}\right.$ $\left.\mathrm{s}^{-1}\right), v$ is the kinematic viscosity of the electrolyte $\left(1.0 \times 10^{-2}\right.$ $\mathrm{cm}^{-2} \mathrm{~s}^{-1}$ ), and $k$ is the electron transfer rate constant. Koutecky-Levich plots for Fe-N-C-900 (Fig. 5d) exhibited good parallel straight lines in the potential range from $-0.3 \mathrm{~V}$ to $-0.7 \mathrm{~V}$, indicating that the ORR on the surface of Fe-N-CS-900 catalyst was a first order reaction to dissolved oxygen concentration in these potential range. ${ }^{24}$ The electron transfer number (n) (Fig. 5e) was calculated from the slopes of Koutecky-Levich plots to be about 4.0 at different potentials, which suggested nearly $4 \mathrm{e}^{-}$reduction process of oxygen. In Tafel plots (Fig. $5 \mathrm{f}$ ), Fe-N-CS-900 had a Tafel slop of $84 \mathrm{mV}$ per decade at low overpotentials, and that of $\mathrm{Pt} / \mathrm{C}$ catalyst was $92 \mathrm{mV}$ per decade. Their similar Tafel slops and close kinetic current densities suggested they had the similar reaction mechanism of ORR on the catalyst 
surface, which verified the superior ORR catalytic property of Fe-N-CS-900. The electrocatalytic stability and methanoltolerant property of $\mathrm{Fe}-\mathrm{N}-\mathrm{CS}-900$ in alkaline media were investigated using the chronoamperometric method. The $i / i_{0}$ shown in Fig. $5 \mathrm{~g}$ were based on chronoamperometric responses for the ORR on $\mathrm{Fe}-\mathrm{N}-\mathrm{CS}-900$ catalyst and commercial $\mathrm{Pt} / \mathrm{C}$ catalyst modified electrodes (Fig. S8a $\uparrow$ ). It is clearly shown that the relative current densities of $\mathrm{Fe}-\mathrm{N}-\mathrm{CS}-900$ retained 95.1\% after 20000 s reaction; however, commercial Pt/C catalyst retained $89.4 \%$. This result indicated that Fe-N-CS-900 had higher stability than commercial Pt/C catalyst. The methanoltolerance property also was investigated. As shown in Fig. 5h, when methanol were added to the electrolytes at $300 \mathrm{~s}$, the current density of commercial Pt/C catalyst had sharp decrease, because its electrocatalytic activity towards methanol oxidation leaded to decrease of current density. In contrast, $\mathrm{Fe}-\mathrm{N}-\mathrm{CS}-900$ catalyst had no significant change, suggesting that Fe-N-CS-900 catalyst had better methanol tolerance than commercial Pt/C catalyst in alkaline media.

To further investigate ORR active sites, we prepared N-doping carbon spheres (N-CS-900) without doping iron element for comparison. Precious works reported two different models to describe the active sites in $\mathrm{M}-\mathrm{N} / \mathrm{C}$ catalyst for ORR. One is the $\mathrm{M}-\mathrm{N}_{x}$ species, ${ }^{27,29,47}$ and the other is the doping $\mathrm{N}$ atoms in the carbon surface. ${ }^{48-50}$ In our control experiment (Fig. 5b), the halfwave potential of N-CS-900 was more negative than that of $\mathrm{Fe}-$ $\mathrm{N}-\mathrm{CS}-900$ in alkaline media, although $\mathrm{N}-\mathrm{CS}-900$ had a high $\mathrm{N}$ content (6.14\%) than that of Fe-N-CS-900. Therefore the Fe- $\mathrm{N}_{x}$ species would act as active sites in alkaline media.

The ORR catalytic behaviour has also been investigated in acidic media. ORR activities of Fe-N-CS-700, 800, 900 and 1000 were shown in Fig. S7, $\uparrow$ and Fe-N-CS-900 exhibited the best ORR activity with the most positive half-wave potential and the largest current density. Furthermore, Fig. 6a showed Fe-N-CS-900 had significantly high ORR activity and it half-wave potential was only $80 \mathrm{mV}$ negative than that of Pt/C catalyst; besides, the limiting current density of $\mathrm{Fe}-\mathrm{N}-\mathrm{CS}-900$ was comparable to that of commercial Pt/C catalyst. The corresponding $\mathrm{K}-\mathrm{L}$ plots for $\mathrm{Fe}-$ N-CS-900 also exhibited the good linearity and the electron transfer number was calculated to be around 4 at different potential range, meaning that $\mathrm{Fe}-\mathrm{N}-\mathrm{CS}-900$ also catalysed a $4 \mathrm{e}^{-}$ oxygen reduction process in $0.5 \mathrm{M} \mathrm{H}_{2} \mathrm{SO}_{4}$ solution. The $i / i_{0}$ in Fig. 6e were based on chronoamperometric responses (Fig. S8b†) for the ORR on Fe-N-CS-900 catalyst and commercial Pt/C catalyst modified electrodes. It was shown that $\mathrm{Fe}-\mathrm{N}-\mathrm{CS}-900$ exhibited higher stability for ORR than commercial Pt/C catalyst, as revealed by relatively lower attenuation rate of current density than Pt/C catalyst. Fig. 6f suggested that Fe-N-CS-900 catalyst had also better methanol tolerance than $\mathrm{Pt} / \mathrm{C}$ catalyst in acidic media. It is noted that the N-CS-900 catalyst exhibited much lower ORR activity than that of Fe-N-CS-900 (Fig. 6a), indicating again that the presence of iron played an important role to the $\mathrm{N}$-doped carbon spheres for ORR in acidic media.

To confirm the significance of hierarchically mesoporous structure on the ORR performance of NPM catalysts, we compared the ORR performance of $\mathrm{Fe}-\mathrm{N}-\mathrm{CS} / \mathrm{SiO}_{2}-900$ with $\mathrm{Fe}-\mathrm{N}-$ CS-900 in both alkaline and acidic media. The electrocatalytic
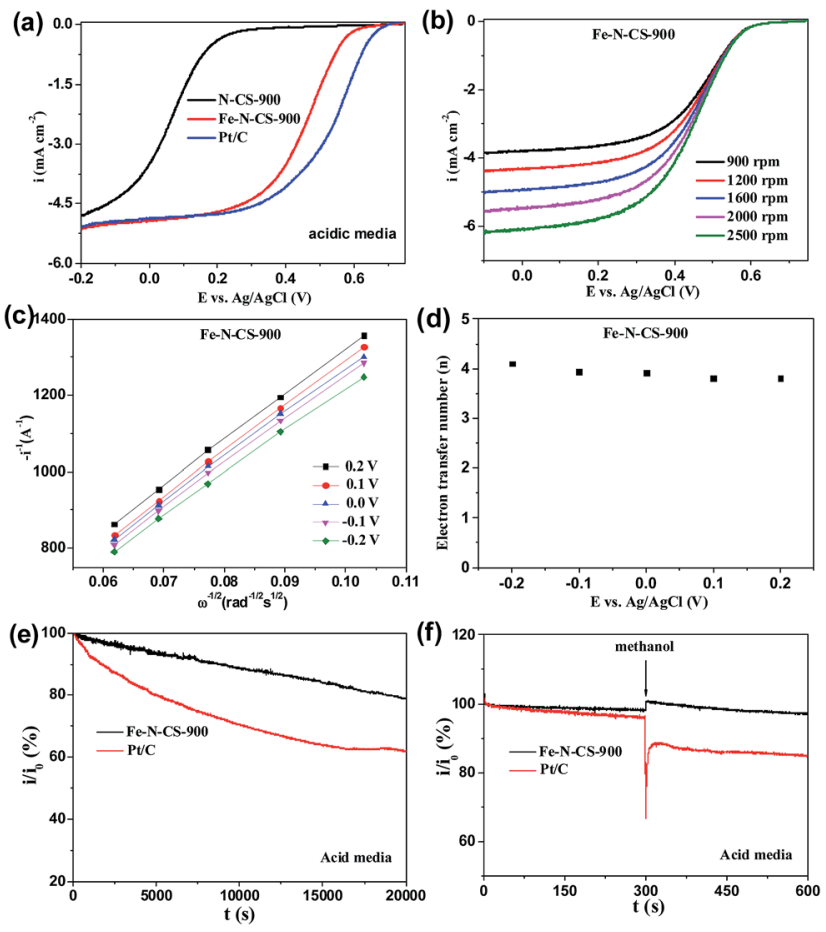

Fig. 6 (a) LSV curves of N-CS-900, Fe-N-CS-900 and Pt/C catalyst modified electrodes at rotation rate of $1600 \mathrm{rpm}$ in $\mathrm{O}_{2}$-saturated acidic media. (b) LSV curves for Fe-N-CS-900 catalyst at different rotation rate. (c) $\mathrm{K}-\mathrm{L}$ plots and (d) the corresponding electron transfer numbers for $\mathrm{Fe}-\mathrm{N}-\mathrm{CS}-900$ catalysts at different electrode potentials. The $i / i_{0}$ were based on chronoamperometric responses for the ORR on the Fe-N-CS-900 catalyst and commercial Pt/C catalyst modified electrodes, (e) in $\mathrm{O}_{2}$-saturated acidic media for $20000 \mathrm{~s}$, (f) with $1 \mathrm{M}$ methanol added at around $300 \mathrm{~s}$ at $0.3 \mathrm{~V}$ in $\mathrm{O}_{2}$-saturated acidic media.
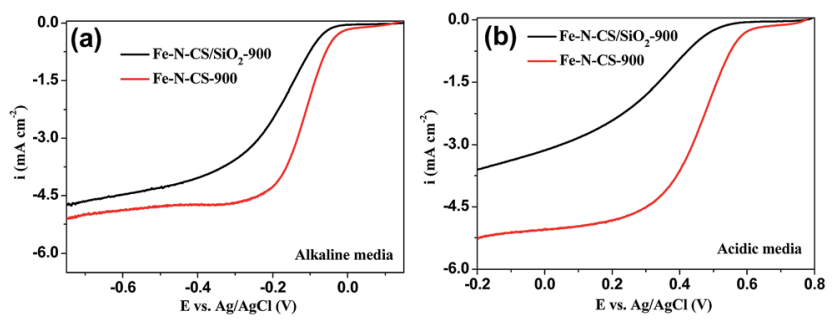

Fig. 7 LSV curves of Fe-N-CS/SiO $2-900$ and $\mathrm{Fe}-\mathrm{N}-\mathrm{CS}-900$ catalysts modified electrodes at rotation rate of $1600 \mathrm{rpm}$ in $\mathrm{O}_{2}$-saturated (a) alkaline media and (b) acidic media, respectively.

study (Fig. 7) showed that $\mathrm{Fe}-\mathrm{N}-\mathrm{CS} / \mathrm{SiO}_{2}-900$ catalyst had the lower ORR activity than that of Fe-N-CS-900 in both alkaline and acidic media. These results indicated that well-developed hierarchically mesoporous structure with high surface area could facilitate the exposure of active sites and benefit the mass-transfer of the reactants and products, leading to higher catalytic activity.

\section{Conclusions}

In this work, we fabricated Fe and $\mathrm{N}$ co-doping hierarchically mesoporous carbon spheres by nanocasting method. The 
obtained catalyst (Fe-N-CS-900) had high surface area $\left(758 \mathrm{~m}^{2}\right.$ $\mathrm{g}^{-1}$ ) and hierarchically mesoporous structure (2 nm, $\left.25 \mathrm{~nm}\right)$. It is demonstrated that Fe-N-CS-900 exhibited high ORR performance in both alkaline and acidic media. We attributed highly efficient catalytic activity to the well-defined hierarchically mesoporous carbon structure with high surface area and homogeneous distribution of iron-nitrogen active sites. In addition, Fe-N-CS-900 also exhibited higher stability, better methanol tolerance and favoured efficient four electron transfer process in both alkaline and acidic media. The iron and nitrogen co-doped hierarchically mesoporous carbon spheres could have potential applications in PEMFCs and metal air batteries.

\section{Acknowledgements}

This work was supported by NSFC (no. 21373116, 21421001 and 21534005), the Tianjin Natural Science Research Fund (13JCYBJC18300), RFDP (20120031110005), and the MOE Innovation Team (IRT13022) of China.

\section{Notes and references}

1 G. Wu and P. Zelenay, Acc. Chem. Res., 2013, 46, 1878.

2 F. Cheng and J. Chen, Chem. Soc. Rev., 2012, 41, 2172.

3 J. Wu and H. Yang, Acc. Chem. Res., 2013, 46, 1848.

4 Y. Bing, H. Liu, L. Zhang, D. Ghosh and J. Zhang, Chem. Soc. Rev., 2010, 39, 2184.

5 X. J. Sun, Y. W. Zhang, P. Song, J. Pan, L. Zhuang, W. L. Xu and W. Xing, ACS Catal., 2013, 3, 1726.

6 D.-S. Yang, D. Bhattacharjya, S. Inamdar, J. Park and J.-S. Yu, J. Am. Chem. Soc., 2012, 134, 16127.

7 Z. Yang, Z. Yao, G. Li, G. Fang, H. Nie, Z. Liu, X. Zhou, X. a. Chen and S. Huang, ACS Nano, 2011, 6, 205.

8 S. Yang, L. Zhi, K. Tang, X. Feng, J. Maier and K. Müllen, Adv. Funct. Mater., 2012, 22, 3634.

9 L. Yang, S. Jiang, Y. Zhao, L. Zhu, S. Chen, X. Wang, Q. Wu, J. Ma, Y. Ma and Z. Hu, Angew. Chem., Int. Ed., 2011, 50, 7132.

10 Z. Yao, H. Nie, Z. Yang, X. Zhou, Z. Liu and S. Huang, Chem. Commun., 2012, 48, 1027.

11 N. Daems, X. Sheng, I. Vankelecom and P. Pescarmona, J. Mater. Chem. A, 2014, 2, 4085.

12 M. Wu, J. Qiao, Y. Liu, J. Zhang, X. J. Zhou and K. X. Li, Green Chem., 2016, 18, 2699.

13 Z. Wu, R. Liu, J. Wang, J. Zhu, W. P. Xiao, C. J. Xuan, W. Lei and D. L. Wang, Nanoscale, 2016, 8, 19086.

14 X. Chen, X. Chen, X. Xu, Z. Yang, Z. Liu, L. Zhang, X. Xu, Y. Chen and S. Huang, Nanoscale, 2014, 6, 13740.

15 K. Ding, Q. Liu, Y. Bu, Y. Huang, J. Lv, J. Wu, S. C. Abbas and Y. Wang, RSC Adv., 2016, 6, 93318.

16 Y.-L. Liu, C.-X. Shi, X.-Y. Xu, P.-C. Sun and T.-H. Chen, J. Power Sources, 2015, 283, 389.

17 K. P. Gong, F. Du, Z. H. Xia, M. Durstock and L. M. Dai, Science, 2009, 323, 760.

18 L. Qu, Y. Liu, J.-B. Baek and L. Dai, ACS Nano, 2010, 4, 1321.

19 R. Liu, D. Wu, X. Feng and K. Müllen, Angew. Chem., Int. Ed., 2010, 49, 2565.
20 T. Y. Ma, Y. Zheng, S. Dai, M. Jaroniec and S. Z. Qiao, J. Mater. Chem. A, 2014, 2, 8676.

21 Y. Liang, H. Wang, J. Zhou, Y. Li, J. Wang, T. Regier and H. Dai, J. Am. Chem. Soc., 2012, 134, 3517.

22 S. Guo, S. Zhang, L. Wu and S. Sun, Angew. Chem., Int. Ed., 2012, 51, 11770.

23 Y. Liang, H. Wang, P. Diao, W. Chang, G. Hong, Y. Li, M. Gong, L. Xie, J. Zhou, J. Wang, T. Z. Regier, F. Wei and H. Dai, J. Am. Chem. Soc., 2012, 134, 15849.

24 Y. Liang, Y. Li, H. Wang, J. Zhou, J. Wang, T. Regier and H. Dai, Nat. Mater., 2011, 10, 780.

25 N. Daems, X. Sheng, I. F. J. Vankelecom and P. P. Pescarmona, J. Mater. Chem. A, 2014, 2, 4085.

26 G. Liu, X. Li, P. Ganesan and B. N. Popov, Electrochim. Acta, 2010, 55, 2853.

27 M. Lefèvre, E. Proietti, F. Jaouen and J.-P. Dodelet, Science, 2009, 324, 71.

28 Y. Zhao, K. Watanabe and K. Hashimoto, J. Am. Chem. Soc., 2012, 134, 19528.

29 L. Lin, Q. Zhu and A.-W. Xu, J. Am. Chem. Soc., 2014, 136, 11027.

30 W. Niu, L. Li, X. Liu, N. Wang, J. Liu, W. Zhou, Z. Tang and S. Chen, J. Am. Chem. Soc., 2015, 137, 5555.

31 M. Li, X. Bo, Y. Zhang, C. Han, N. Anaclet and L. Guo, J. Mater. Chem. A, 2014, 2, 11672.

32 F. Jaouen, E. Proietti, M. Lefevre, R. Chenitz, J.-P. Dodelet, G. Wu, H. T. Chung, C. M. Johnston and P. Zelenay, Energy Environ. Sci., 2011, 4, 114.

33 J. C. Li, S. Y. Zhao, P. X. Hou, R. P. Fang, C. Liu, J. Liang, J. Luan, X. Y. Shan and H. M. Cheng, Nanoscale, 2015, 7, 19201.

34 H.-W. Liang, W. Wei, Z.-S. Wu, X. Feng and K. Müllen, J. Am. Chem. Soc., 2013, 135, 16002.

35 A. Kong, B. Dong, X. Zhu, Y. Kong, J. Zhang and Y. Shan, Chem.-Eur. J., 2013, 19, 16170.

36 J. Y. Cheon, T. Kim, Y. Choi, H. Y. Jeong, M. G. Kim, Y. J. Sa, J. Kim, Z. Lee, T.-H. Yang, K. Kwon, O. Terasaki, G.-G. Park, R. R. Adzic and S. H. Joo, Sci. Rep., 2013, 3, 2715.

37 A. Kong, Y. Kong, X. Zhu, Z. Han and Y. Shan, Carbon, 2014, 78, 49.

38 A. Kong, X. Zhu, Z. Han, Y. Yu, Y. Zhang, B. Dong and Y. Shan, ACS Catal., 2014, 4, 1793.

39 C. Deng, H. Zhong, L. Yao, S. Liu, Z. Xu and H. Zhang, ChemSusChem, 2014, 7, 3435.

40 Y. Liu, X.-J. Jin, A.-X. Tuo and H. Liu, $R S C A d v ., 2016,6$, 105211.

41 J.-G. Wang, H.-J. Zhou, P.-C. Sun, D.-T. Ding and T.-H. Chen, Chem. Mater., 2010, 22, 3829.

42 S. Chen, J. Bi, Y. Zhao, L. Yang, C. Zhang, Y. Ma, Q. Wu, X. Wang and Z. Hu, Adv. Mater., 2012, 24, 5593.

43 R. Arrigo, M. Hävecker, R. Schlögl and D. S. Su, Chem. Commun., 2008, 4891.

44 W. Yang, T.-P. Fellinger and M. Antonietti, J. Am. Chem. Soc., 2010, 133, 206.

45 J. Liang, Y. Zheng, J. Chen, J. Liu, D. Hulicova-Jurcakova, M. Jaroniec and S. Z. Qiao, Angew. Chem., Int. Ed., 2012, 51, 3892. 
46 X. J. Sun, P. Song, Y. W. Zhang, C. P. Liu, W. L. Xu and W. Xing, Sci. Rep., 2013, 3, 2505.

47 S. Zhang, B. Liu and S. Chen, Phys. Chem. Chem. Phys., 2013, 15, 18482.

48 R. Silva, D. Voiry, M. Chhowalla and T. Asefa, J. Am. Chem. Soc., 2013, 135, 7823.
49 R. Wang, T. Zhou, H. Wang, H. Feng and S. Ji, J. Power Sources, 2014, 269, 54.

50 S. Kundu, T. C. Nagaiah, W. Xia, Y. Wang, S. V. Dommele, J. H. Bitter, M. Santa, G. Grundmeier, M. Bron, W. Schuhmann and M. Muhler, J. Phys. Chem. C, 2009, 113, 14302. 